\title{
Ecuación del flujo gradualmente variado en canales rectangulares de fondo curvo
}

\author{
G. Sotelo-Ávila y J. Gallegos-Silva. \\ Departamento de Hidráulica. División de Ingeniería Civil, Topográfica y Geodésica \\ Facultad de Ingeniería, UNAM \\ E-mail:soteloa@servidor.unam.mx.
}

(recibido: julio de 2001; aceptado: octubre de 2001)

\begin{abstract}
Resumen
Las hipótesis del movimiento rectilíneo y de distribución hidrostática de la presión son ciertamente las más importantes en la hidráulica de canales, y de ellas se derivan los principales modelos de flujo que usualmente se emplean. Sin embargo, no es válido aplicar las mismas hipótesis y métodos de análisis al flujo curvilíneo, que ocurre cuando el canal adopta curvaturas verticales en el fondo, las cuales inducen cambios importantes en la distribución de la velocidad, presión y hasta en la pérdida de energía. Tal es el caso de canales que contienen curvas verticales para unir tramos de distinta pendiente y producir el cambio en la dirección del flujo en cubetas deflectoras y vertedores en túnel.

Los autores de este artículo proponen una ecuación de flujo gradualmente variado en canales rectangulares de fondo curvo, ésta es de gran utilidad en la determinación del perfil del flujo con dichas características, donde se plantea la adición de un factor de amplificación de la velocidad en el cálculo del gradiente local de fricción, para tomar en cuenta el efecto de la distribución de la velocidad en el flujo curvilíneo.

Con estos resultados se considera factible. analizar perfiles de flujo en un cimacio, o en canales con curvas verticales donde se han utilizado, hasta ahora, métodos convencionales de análisis basados en las teorías del movimiento rectilíneo.
\end{abstract}

Descriptores: flujo curvilíneo, perfiles de flujo gradualmente variado, factor de amplificación, gradiente local de fricción.

\section{Abstract}

The study of channel flow usually have its basis in the hydrostatic distribution of pressure and the rectilinean flow hypotheses. It is from this hypothesis that the main flow equations are obtained. However, this is not applicable to a vertically curved flow that is present in a curved bed channel. This kind of channel is used to join two different slopes or in ski jumps. This kind of flow presents several changes from the rectilinean flow as in the velocity and pressure distributions and even in the energy loses.

The authors of this article propose an equation of gradually varied flow for vertically-curved bed rectangular channels that adds a coefficient to modify the velocity in the calculus of the local friction gradient.

With these results is possible now to analyze flow profiles in vertically-curved bed channels where before were used the methods for straight channels and therefore, increase accuracy. 


\section{Introducción}

Sotelo y Ruíz (1994) demostraron la forma de determinar la energía específica en un canal rectangular de fondo curvo, tomando como hipótesis la ley del vórtice libre para la distribución de la velocidad, ya que se inducen líneas de corriente de gran curvatura y variación de la presión con respecto de la hidrostática. Obtuvieron la ecuación

$$
E=d \cos \theta+\frac{1}{\lambda^{2}} \frac{V^{2}}{2 g}=d \cos \theta+\frac{1}{\lambda^{2}} \frac{Q^{2}}{A^{2} 2 g}
$$

donde $\mathrm{V}=\frac{\mathrm{Q}}{\mathrm{A}}$ es la velocidad media en una sección ortogonal al fondo y d el tirante en la sección.

También encontraron que la velocidad media es

$$
\mathrm{V}=\lambda v_{a}
$$

donde

$$
\lambda=\left[1-\frac{R}{d}\right] \ln \left[1-\frac{d}{R}\right]=\left[\frac{1-\kappa d}{\kappa d}\right] \ln [1-\kappa d]
$$

es el coeficiente de curvatura y $v_{a}$ la velocidad al nivel de la superficie libre.

E energía específica respecto del fondo del canal en una sección determinada.

$\theta$ ángulo que forma la tangente a la plantilla del canal con respecto de la horizontal en dicha sección.

$\mathrm{R}$ radio de curvatura del fondo

El coeficiente de curvatura $\lambda$ corrige el efecto de considerar la velocidad media (V) en la sección, en lugar de la velocidad sobre la superficie libre del agua $\left(v_{a}\right), \kappa=1 /$ R es la curvatura del fondo del canal y $d$ el tirante perpendicular a dicho fondo. Cuando el flujo es convexo la curvatura $\kappa<0$ y los términos que contienen a $\kappa d$ cambian de signo en la ecuación (3).

Un hecho importante es que para calcular la energía en una sección del flujo con curvatura vertical, no es necesario conocer la distribución de la presión, ni la que hay en el fondo, sólo el coeficiente de curvatura $\lambda$. Cuando $\kappa d$ es pequeño $(<0.1) \circ \mathrm{el}$ flujo es rectilíneo $\lambda=1$, la ecuación (1) se convierte en la expresión convencional para el líquido ideal.

En efecto, con frecuencia es necesario conocer la distribución de la presión en la sección del flujo con curvatura vertical y sobre todo, la que hay en el fondo. Dicha distribución es fácil de obtener si se establece que la energía es la misma en un punto cualquiera de la sección, a la altura $z=t \cos \theta$, y otro sobre la superficie libre $(z=d \cos \theta)$ en la forma

$$
t \cos \theta+\frac{p}{g \rho}+\frac{v^{2}}{2 g}=d \cos \theta+\frac{v_{a}^{2}}{2 g}
$$

si $d^{\prime}=d-t$, donde $t$ es la distancia del fondo al punto, la presión en cualquier punto es

$$
\frac{p}{g \rho}=d^{\prime} \cos \theta+\left[1-\left(\frac{v}{v_{a}}\right)^{2}\right] \frac{v_{a}^{2}}{2 g}
$$

pero $y / v_{a}=r_{a} / r$, y con la ecuación (2) se obtiene

$$
\frac{p}{g \rho}=d^{\prime} \cos \theta+\left[1-\left(\frac{r_{a}}{r}\right)^{2}\right] \frac{1}{\lambda^{2}} \frac{V^{2}}{2 g}
$$

expresión que permite conocer la presión en cualquier punto de la sección, de radio $r$, sea el flujo cóncavo o convexo. Para el cóncavo, cuando $r=R, r_{a}=R-d$ y $d^{\prime}=d$, se obtiene la carga de presión en el fondo

$$
\frac{p_{b}}{g \rho}=d \cos \theta+\left[2 \frac{d}{R}-\left(\frac{d}{R}\right)^{2}\right] \frac{1}{\lambda^{2}} \frac{V^{2}}{2 g}
$$

Para el flujo convexo, $r_{a}=\mathrm{R}+d$ y el signo del último término de la ecuación cambia a negativo, por lo que se usa el doble signo como sigue

$$
\frac{p_{b}}{g \rho}=d \cos \theta \pm\left[\frac{1-(d / 2 R)}{\lambda^{2}}\right] \frac{V^{2} d}{g R}
$$

si $\kappa d \leq 0.1, \lambda=1.0$, y la ecuación anterior se simplifica a la forma

$$
\frac{p_{b}}{g \rho}=d \cos \theta \pm \frac{V^{2} d}{g R}
$$


El primer término en las dos últimas ecuaciones corresponde a la carga de presión hidrostática y el segundo a la desviación de la presión por efecto de la curvatura de las líneas de flujo. Dicha desviación se agrega a la hidrostática cuando el flujo es cóncavo, resultando siempre presiones positivas. La presión en flujo convexo disminuye a valores que pueden ser menores que la atmosférica.

Según la ecuación (1), la carga de presión en el fondo no interviene en la magnitud de la energía.

Por ejemplo, para el fondo de la sección resulta

$$
\mathrm{E}=\frac{p_{b}}{g \rho}+\frac{v_{b}^{2}}{2 g}
$$

pero $\frac{p_{b}}{\gamma}$ se obtiene de la ecuación (7), y $v_{b}=\frac{r_{a} V}{\lambda R}$, cuya sustitución en la ecuación de la energía específica E lleva nuevamente a la ecuación (1).

Sotelo y Gallegos (2000) también presentaron una aportación al cálculo del régimen crítico en un canal rectangular, donde demostraron que la derivada de la energía específica adopta la forma

$$
\frac{d E}{d d}=\cos \theta-\frac{q^{2}}{d^{3} \lambda^{3} g}\left[\frac{(\kappa d-1)+\kappa \lambda d}{(\kappa d-1)}\right]
$$

donde $q$ es el gasto unitario en el canal.

\section{Desarrollo}

Ecuación dinámica del flujo gradualmente variado en canales rectangulares de fondo curvo

La ecuación diferencial de la energía para un flujo unidimensional permanente en un canal de fondo curvo es

$$
\frac{d}{d s}\left(z+d \cos \theta+\frac{1}{\lambda^{2}} \frac{V^{2}}{2 g}+h_{f}\right)=0
$$

o bien

$$
\frac{d z}{d s}+\frac{d E}{d s}+\frac{d h_{f}}{d s}=0
$$

siendo:

$$
-\frac{d z}{d s}=\operatorname{sen} \theta=S_{o}
$$

la pendiente local de la plantilla del canal ( si se considera que $z$ decrece en la dirección s), $\frac{d \mathrm{E}}{d s}=\frac{d \mathrm{E}}{d d} \frac{d d}{d s}$ y además $\frac{d h_{f}}{d s}=S_{f}$; por lo que al sustituir en la ecuación (11)

$$
\frac{d d}{d s}=\frac{S_{0}-S_{f}}{\frac{d E}{d d}}
$$

que representa la ecuación dinámica del flujo gradualmente variado para canales de fondo curvo, donde $d E$ / $d d$ viene expresada por la ecuación (9) para canales rectangulares.

Por otra parte, para tomar en cuenta la resistencia al flujo en un canal rugoso y la acción de vórtices producto de la turbulencia, se sigue el camino usual de introducir un término empírico en la ecuación del momentum, como se hace al derivar la ecuación de Chezy. Para ello, se considera un volumen de control en forma de cuña delgada como se muestra en la figura 1 , con fondo curvo $\mathrm{K}(\mathrm{s})>0$ y para el instante $t=t_{0}$.

La fuerza de resistencia externa tangente al fondo de la cuña en la dirección negativa de s vale

$$
\mathrm{F}_{0}=-\mathrm{K} \rho v_{0}^{2} \sigma d s
$$

donde $\mathrm{K}$ es un factor adimensional (empírico) de proporcionalidad de la masa de líquido contenido en la cuña y el cuadrado de la velocidad en el fondo. Dicho factor depende de parámetros que miden la rugosidad de la frontera y es función también de la curvatura del fondo, $v_{0}$ es la velocidad local en el fondo.

De manera similar, la fuerza de resistencia en las paredes es

$$
F_{w}=-K \rho \int_{n=0}^{d} v^{2}(n) d a d n
$$

donde da es la diferencial de arco a la distancia $n$, cuando hay un incremento diferencial (ds) en el fondo, es decir, $d a=(1-\kappa n) d s$. Lo que resulta entonces

$$
F_{w}=2 K \rho v_{0}^{2} \frac{\ln (1-\kappa d)}{\kappa} d s
$$



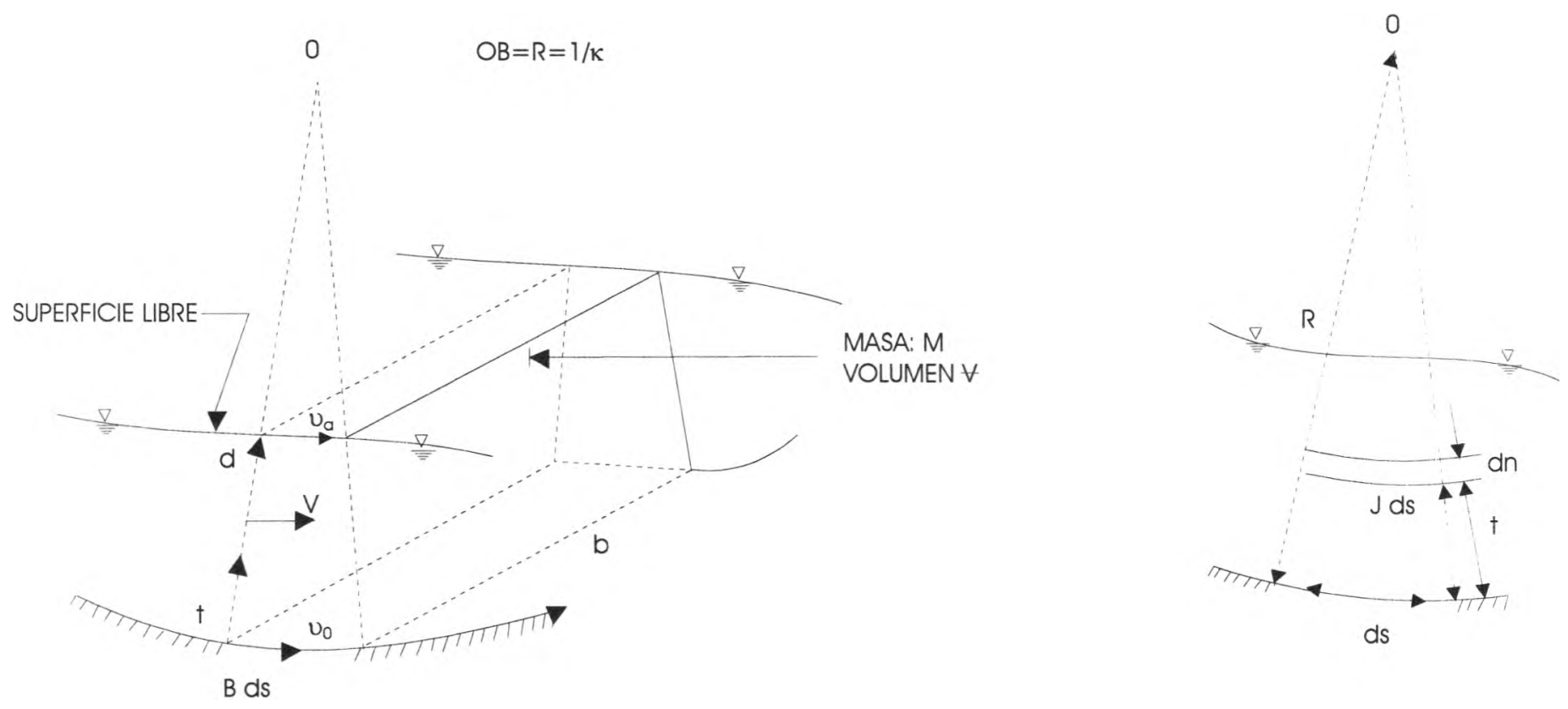

Figura 1. Volumen de control para obtener las ecuaciones de fricción de Dressler en un canal rectangular de fondo cóncavo $(K>0)$

al sumar $F_{0}$ y $F_{w}$, la fuerza total sobre la masa es

$$
F_{t}=-K \rho v_{0}^{2}\left[1-2 \frac{\ln (1-\kappa d)}{\kappa b}\right] b d s
$$

Al simplificar la masa contenida en el volumen del elemento de control $m=\rho b(d) d$ s se obtiene la fuerza total de resistencia ejercida por unidad de masa y se considera como una fuerza de cuerpo uniformemente distribuida en dicha masa. Su magnitud es

$$
\mathrm{F}_{r}=\frac{\mathrm{F}_{t}}{m}=-\mathrm{K} \frac{v_{0}^{2}}{\left[\frac{d}{\left.1-2 \frac{\ln (1-\kappa d)}{\kappa b}\right]}\right.}
$$

Si se hace una analogía con el desarrollo que se utiliza para obtener la ecuación de Chezy del flujo rectilíneo, el esfuerzo tangencial medio de resistencia se considera $\tau_{0}=\mathrm{K}_{\rho} \mathrm{V}^{2}$, donde $\mathrm{V}$ es la velocidad media del flujo, de manera que la fuerza total de resistencia se expresa como

$$
F_{t}=-\tau_{0} P d s=-K \rho V^{2} P d s
$$

Siendo $\rho \mathrm{A}$ da la masa del elemento y $\mathrm{R}_{h}=\mathrm{A} / \mathrm{P}$ el radio hidráulico de la sección, la fuerza de resistencia por unidad de masa vale

$$
F_{r}=-K \frac{V^{2}}{R_{h}}
$$

Además, con $\tau_{0}=K \rho V^{2}=g \rho R_{h} S_{f}, K V^{2}=g R_{h} S_{f}$, la anterior resulta

$$
F_{r}=-g S_{f}
$$

donde $S_{f}$ representa el gradiente local de fricción.

Al comparar las ecuaciones (17) y (18) se observa que el radio hidráulico del flujo aquí tratado se interpreta como el término dentro del paréntesis rectangular en la primera ecuación, al cual puede 
designarse como radio hidráulico efectivo, en la forma

$$
R_{h e}(b, d, k)=\frac{d}{1-2 \frac{\ln (1-\kappa d)}{\kappa b}}
$$

El mismo resultado se obtiene cuando $\kappa<0$. También, cuando $(-\kappa) \rightarrow 0, \ln (1-\kappa d) \rightarrow-\kappa d, R_{h e}=R_{h}$ $=6 d /(6+2 d)$, es decir, la ecuación (17) coincide con la ecuación (18.a) para un fondo plano.

$\operatorname{De}_{h}=6 d /(6+2 d)$, resulta $d=(1+2 d / 6) R_{h} y$ los términos restantes de la ecuación (19) se convierten en

$$
\frac{1-2 \frac{\ln (1-\kappa d)}{\kappa b}}{d}=\left[\frac{1-2 \frac{\ln (1-\kappa d)}{\kappa b}}{1+(2 d / b)}\right] \frac{1}{R_{h}}
$$

donde se introdujo el radio hidráulico convencional $\mathrm{R}_{h}$ de la sección.

El término

$$
a_{v}=\left[\frac{1-2 \frac{\ln (1-\kappa d)}{\kappa b}}{1+(2 d / 6)}\right]
$$

se conoce como factor de amplificación de la velocidad, ya que mide el incremento o decremento necesario de $v_{0}^{2}$ para generar la misma fuerza de resistencia que la velocidad variable $v$.

Por otra parte, cuando se utiliza la ecuación de Chezy para la fricción o la correspondiente a Manning, el valor de K se expresa como

$$
\mathrm{K}=\frac{g}{C^{2}}=\frac{g n_{M}^{2}}{R_{h}^{1 / 3}}
$$

donde se ha utilizado la equivalencia $C=R_{h}{ }^{1 / 6} / n_{M}$, siendo $C$ el coeficiente de Chezy y $n_{M}$ el coeficiente de Manning. Si el fondo se comporta como pared hidráulicamente de transición, es suficiente emplear equivalencias similares de C, como la expresada por la ecuación de Colebrook-White.

Al sustituir K de la ecuación anterior en la (17), la fuerza total de resistencia por unidad de masa se reduce a

$$
\mathrm{F}_{r}=-g a_{v} \frac{v_{0}^{2}}{\mathrm{C}^{2} \mathrm{R}_{h}}=-g a_{v} \frac{n_{\mathrm{M}}^{2} v_{0}^{2}}{\mathrm{R}_{h}^{4 / 3}}
$$

la pendiente local de fricción en la ecuación (12) valdrá entonces

$$
s_{f}=a_{v} \frac{v_{0}^{2}}{C^{2} R_{h}}=a_{v} \frac{n_{M}^{2} v_{0}^{2}}{R_{h}^{4 / 3}}
$$

Con la ecuación (12) y los resultados obtenidos en las expresiones (9) y (22) es posible analizar los perfiles de flujo gradualmente variado en canales rectangulares de fondo curvo. La solución es por ello numérica, empleando métodos similares a los que se usan con la ecuación convencional.

Por último, se puede aplicar la ecuación de la energía entre dos secciones en canales con las características anteriores, se tendrá que

$z_{1}+d_{1} \cos \theta_{1}+\frac{1}{\lambda_{1}^{2}} \frac{V_{1}^{2}}{2 g}=z_{2}+d_{2} \cos \theta_{2}+\frac{1}{\lambda_{2}^{2}} \frac{V_{2}^{2}}{2 g}+s_{1} \Delta s(23)$

por medio de la cual es posible calcular el perfil del flujo entre ambas secciones, de la misma forma que se sigue con el método estándar por pasos.

\section{Conclusiones}

El análisis del flujo gradualmente variado en un canal de fondo curvo se realiza integrando las ecuaciones (12) y (9) por métodos convencionales (directo por pasos, estándar por pasos, Euler, Runge-Kutta de cuarto orden, etc.). La pendiente de fricción se calcula con la ecuación (22) donde figura el factor de amplificación $a_{\nu}$ que toma en cuenta la distribución de la velocidad en la sección del flujo curvilíneo y se expresa mediante la ecuación (20).

La ecuación (23) es válida en canales prismáticos o no prismáticos de fondo curvo y se diferencia de la empleada para el movimiento rectilíneo en el coeficiente $\lambda$ de curvatura en cada sección transversal (dado por la ecuación 3) y en la expresión para calcular $\mathrm{S}_{f}$ (ecuación 22). 
Los resultados obtenidos significan que el canal puede tener curvatura variable en su desarrollo, de acuerdo con las curvas verticales que se empleen en la concepción de su geometría.

\section{Referencias}

Sotelo G. y Gallegos J. (2000). Régimen crítico en flujo curvilíneo. XIX Congreso Latinoamericano de Hidráulica, Córdoba, Argentina; XVI Congreso Nacional de Hidráulica, Morelia, Mich., México.
Sotelo G. y Ruíz R. (1994). Flujo curvilíneo como un vórtice libre. XIII Congreso Nacional de Hidráulica, Puebla, Puebla. México.

\section{Semblanza de los autores}

Gilberto Sotelo-Ávila. Obtuvo los grados de ingeniero civil, maestro en ingeniería y doctor en ingeniería en la Facultad de Ingeniería de la UNAM. Inició su actividad universitaria en 1958 como investigador en el Instituto de Ingeniería y como profesor en licenciatura y posgrado dentro de la misma facultad, actividades que funge actualmente. Fue jefe de la División de Ingeniería Civil, Topográfica y Geodésica, consejero técnico de la Facultad de Ingeniería y consejero académico de área en la UNAM. Es investigador nacional nivel 2 del Sistema Nacional de Investigadores del CONACYT y ha recibido los premios Universidad Nacional 1998, Docencia en Ciencias Exactas, en la UNAM y el Premio Nacional Miguel A. Urquijo 1999, otorgado por el Colegio de Ingenieros Civiles de México al mejor artículo técnico publicado durante 1997 y 1998. El Consejo Técnico de la Facultad de Ingeniería de la UNAM le otorgó las cátedras especiales Javier Barros Sierra en 1999 y Mariano Hernández Barrenchea en el año 2000. Es miembro de la Academia Mexicana de Ciencias de la Asociación Internacional de Investigaciones Hidráulicas del Colegio de Ingenieros Civiles de México y del Consejo Académico del CENEVAL para el examen de calidad profesional de los ingenieros civiles.

Jesús Gallegos-Silva. Obtuvo el título de Ingeniero Civil en 1987 en la Facultad de Ingeniería de la UNAM y realizó estudios de maestría en la División de Estudios de Posgrado de la Facultad de Ingeniería. Desde 1987 imparte cátedra en el área de ingeniería hidráulica y a la fecha las asignaturas de hidráulica básica, hidráulica de canales, máquinas hidráulicas y transitorios. Ha participado en la revisión, diseño e implementación de nuevas prácticas de laboratorio para las asignaturas obligatorias del Departamento de Hidráulica. Asimismo, el Consejo Técnico de la Facultad de Ingeniería de la UNAM le otorgó las cátedras especiales Enrique Rivero Borrel en 1994 y Aurelio Benassini Vizcaíno en el 2000. Desde 1994 a la fecha es coordinador del Laboratorio de Hidráulica. Ha participado en Congresos de Hidráulica, tanto nacionales como internacionales. 